\title{
Levosulpiride Use in Chronic Kidney Disease Patients - Do We Need to Be Cautious?
}

\author{
Srinivasaprasad.N.D, DM (Nephrology) ${ }^{1}$, Chandramohan.G, DM (Nephrology) ${ }^{2}$ \\ Brinda Srinivasagopalane, MD (Physiology) ${ }^{3}$, \\ Edwin Fernando M, DM (Nephrology), FRCP (Glas), FRCP (Edin) ${ }^{4}$ \\ ${ }^{1}$ Assistant Professor, Department of Nephrology, Stanley Medical College, Chennai. \\ ${ }^{2}$ Associate professor, Department of Nephrology, Madurai Medical College, Madurai. \\ ${ }^{3}$ Assistant Professor, Department of Physiology, ACS Medical College, Chennai. \\ ${ }^{4}$ Professor \& Head, Department of Nephrology, Stanley Medical College, Chennai.
}

\begin{abstract}
:
Background: A challenging scenario for today's clinician is to treat dyspeptic symptoms among the chronic kidney disease (CKD) patients. Levosulpiride is a substituted benzamide which is most commonly used as a prokinetic drug. At present there is enough evidence to say that levosulpiride causes movement disorders (LIM) when used in elderly patients. This study attracts attention as there was high occurrence of extra pyramidal symptoms (EPS) in CKD patients even with recommended doses for dyspeptic symptoms.
\end{abstract}

Methodology: This study was done in the Department Of Nephrology of a tertiary care private hospital by retrospective analysis of data gathered from patients who had developed movement disorders following exposure to levosulpiride for dyspeptic symptoms. Statistical analysis was done using Microsoft XL sheet.

Results: Twelve patients (nine males and three females) with varying degrees of chronic kidney disease who developed levosulpiride induced movement disorders were included in the study. The number of patients who developed dystonia, Parkinsonism, isolated tremors and Orofacial dyskinesia were eight, two, one, and one $(66.6 \%, 16.6 \%, 8.3 \%$ and $8.3 \%)$ respectively. All except one patient recovered completely after the discontinuation of medication.

Conclusion: Levosulpiride is an effective prokinetic agent. Even though the occurrence of LIM is rare in patients with normal kidney function, LIM occurs much more commonly in chronic kidney disease patients contrary to the belief. Hence the dose of levosulpiride should be modified in patients with chronic kidney disease and prompt discontinuation of levosulpiride should be done with the occurrence of LIM as it leads to complete recovery of symptoms.

Keywords: Chronic kidney disease, Elderly, Levosulpiride, Movement Disorders, Prokinetic agent

\section{Introduction}

A challenging scenario for today's clinician is to treat dyspeptic symptoms among the chronic renal failure patients. One of the commonest causes of dyspepsia among renal compromise patients is high urea levels. The dyspeptic symptoms of nausea and vomiting are fairly common with all stages of renal failure and those on dialysis and post renal transplantation. Over the years, varied drug protocols have emerged and tried to treat dyspepsia.

Levosulpiride is a substituted benzamide which is most commonly used as a prokinetic drug and atypical antipsychotic. The uses of levosulpiride is multiple including: depression, psychosis, somatoform disorders, emesis and dyspepsia (1). The proposed mechanism is that, it blocks the presynaptic dopaminergic D2 receptors. There are several multi-centric trials comparing the superior effects of levosulpiride over other antiemetics and one such study by Corazza et al has recorded significant improvement in dyspepsia with levosulpiride (2). Acting as both a dopaminergic receptor antagonist and a serotonin receptor agonist, it increases gastric and gall bladder emptying (3) .

The most common side effects are amenorrhoea, galactorrhoea and gynaecomastia and it can also cause extrapyramidal symptoms (EPS), most of which are generalized Parkinsonism. Levosulpiride crosses the blood brain barrier (4). This central action of levosulpiride on D2 receptors has been attributed to its effect to cause extrapyramidal symptoms such as dystonia, akathisia, tardive dyskinesia and tremors. But most of those side effects are seen with higher doses of the drug. Levosulpiride is the only agent within this class of substituted benzamides that is used as a neuroleptic agent, although at dosages higher (200-300 mg daily) than those used in gastroenterology ( $25 \mathrm{mg}$ t.d.s.). This is probably the reason why levosulpiride has only a low potential to induce extrapyramidal side-effects at the doses used in gastroenterology. A few authors had previously documented about the movement disorders caused by Levosulpiride. Shin H.W et al stated that until 
recently, the drug-induced movement disorders related to Levosulpiride were under-recognized, but it has now been shown that Levosulpiride frequently causes Parkinsonism (5).

Yet another study by Hyun et al demonstrated levosulpiride associated neurological disturbances such as chorea(6). Usually extrapyramidal symptoms occur more frequently in women than in men and more frequently in children and the elderly than in adults. At present there is enough evidence to say that levosulpiride causes movement disorders when used in elderly patients. To our knowledge there are no case reports in chronic kidney disease patients. This study attracts attention as there was high occurrence of EPS in CKD patients even with recommended doses of levosulpiride for dyspeptic symptoms.

\section{Aim}

To study the movement disorders induced by the prokinetic dose of Levosulpiride.

\section{Methodology}

This study was done in the Department Of Nephrology of a tertiary care private hospital by retrospective analysis of data gathered from patients who had developed movement disorders following exposure to levosulpiride for dyspeptic symptoms. All these patients were in varying stages of CKD. They had received levosulpiride either orally or intravenously at a dose of $25 \mathrm{mg}$ thrice daily half an hour before food intake. Medication was not administered according to weight or renal status of the patient. This group of patients were only receiving antihypertensives, insulin and haematinics along with Levosulpiride. Medications were stopped immediately after the occurrence of neurological manifestations. Patients who presented with dystonia and Orofacial dyskinesia were treated with injection lorazepam at presentation and followed by Tab. Clonazepam $0.25 \mathrm{mg}$ two times a day till the disappearance of symptoms. Those presented with Parkinsonism were treated with Tab.Trihexiphenydyl $2 \mathrm{mg}$ twice a day till the symptoms disappeared. The patients' demographic data like age, sex, diagnosis, estimated GFR (calculated by MDRD study equation), dose of medication, time of onset, type, duration and outcome of movement disorder were studied. The study was cleared by institutional ethics committee.

Statistical analysis was done using Microsoft XL sheet.

\section{Results}

Twelve patients (nine males and three females) with varying degrees of chronic kidney disease who developed levosulpiride induced movement disorders were included in the study. Mean age of the patients was 42 yrs. Mean EGFR of the study population was $23.7 \mathrm{ml} / \mathrm{min} / 1.73 \mathrm{~m} 2$. Eight $(66.6 \%)$ patients received oral medication, two (16.6\%) patients received intravenous (IV) form and two (16.6\%) patients received IV medication followed by oral form. Mean duration from the onset of exposure to levosulpiride to the onset of movement disorders was 6.6 days. Of the 12 patients, four were on maintenance hemodialysis, two were renal transplant recipients. The number of patients who developed dystonia, Parkinsonism, isolated tremors and Orofacial dyskinesia were eight, two, one, and one $(66.6 \%, 16.6 \%, 8.3 \%$ and $8.3 \%)$ respectively. All except one patient recovered completely after the discontinuation of medication. Mean age of patients who developed dystonia was 35.44 yrs. Mean duration of onset of dystonia was three days. Mean duration of onset of recovery for dystonia was 2.99 days.

Patient characteristics are shown in table 1.

\section{Discussion}

This retrospective descriptive study was done in a tertiary care private hospital in Chennai to highlight the common occurrence of movement disorders in chronic kidney disease patients exposed to the prokinetic drug levosulpiride. This study included 12 patients in different age groups with varying stages of CKD. Majority of patients developed dystonia either orolingual or truncal dystonia. Younger patients developed dystonia compared to older patients who developed non dystonic movement disorders. These patients developed dystonia early in the course of treatment. Patients who developed Parkinsonism were older and received Levosulpiride for prolonged periods before the development of symptoms. All the patients had complete recovery after the discontinuation of medication. Patients with Parkinsonism took many weeks to improve.

In a study by Shin et al, among 132 consecutive patients who were diagnosed with drug-induced movement disorders between January 2002 and March 2008, 91 patients with Levosulpiride Induced Movement Disorder (LIM) were identified. Seventy-eight (85.7\%) patients were aged more than 60 years. The most common LIM was levosulpiride induced Parkinsonism (LIP) $(\mathrm{n}=85,93.4 \%)$, followed by tardive dyskinesia ( $\mathrm{n}$ $=9,9.9 \%)$ and isolated tremor $(\mathrm{n}=3,3.3 \%)$. LIM persisted after withdrawal of levosulpiride in $48.1 \%$ of patients with LIP, $66.7 \%$ with dyskinesia, and none with isolated tremor (7). Levosulpiride frequently causes drug-induced movement disorders, presenting mainly with LIP followed by lower face dyskinesia. The symptoms are often severe and irreversible even after the withdrawal of levosulpiride. In contrast to our study, 
this study included patients with normal kidney function. In our study dystonia was the commonest levosulpiride induced movement disorder and all of them had complete recovery with discontinuation of medication.

A similar case series of 3 patients by Diwan et al reported the occurrence of LIM (Dyskinesia-1, LIP-1 \& Dystonia - 1) (8). Like our patients, all these patients developed LIM within short period of exposure to levosulpiride.

For reasons unknown levosulpiride induced dystonia is relatively uncommon in the elderly. Parkinsonism is the commonest LIM in the elderly, because these people are already prone to develop Parkinson's disease. And the incomplete recovery could be due to pre existing Parkinson's disease.

Levosulpiride is the levo enantiomer of Sulpiride. It is a substituted benzamide which is meant to be used for several indications: depression, psychosis, somatoform disorders, emesis and dyspepsia. The levo enantiomer shows better pharmacological actions and lower incidence of toxic effects than both dextro and the racemic forms of the drug (1). Levosulpiride is an atypical antipsychotic agent (stimulating activity al low doses and sedative effects at high doses) that blocks the presynaptic dopaminergic D2 receptors. Like its parent compound, levosulpiride shows antagonism at D3 and D2 receptors present presynaptically as well as postsynaptically (1). It has moderate partial 5-HT4 receptor agonist properties and an extremely weak 5-HT3 antagonism. This property of levosulpiride enhances its therapeutic efficacy as a prokinetic agent in the gastrointestinal tract (9). The oral bioavailability of levosulpiride is about 30\% with peak plasma concentrations achieved at about 3 hrs. Its plasma half-life is $9.7 \mathrm{hrs}$ (oral); $4.3 \mathrm{hrs}$ (IV). It is $40 \%$ protein bound. It is mainly excreted via urine (90-95\%). In a multi-center, double blind controlled trial enrolling 1298 patients with functional dyspepsia; levosulpiride was more effective than domperidone, metoclopramide and placebo. In a small study on 30 patients with functional dyspepsia and gastroparesis, levosulpiride was found to be as effective as cisapride. All prokinetics with D2 receptor antagonist properties have been found to induce extrapyramidal reactions. Extrapyramidal reactions by neuroleptics depend on the affinity values for the D2 receptor meaning antipsychotics with low dissociation constants bind tightly to dopamine D2 receptor and readily elicit extrapyramidal symptoms. But levosulpiride has high dissociation constant meaning it binds loosely to D2 receptors and elicits only minimal extrapyramidal side effects (9). Levosulpiride only at high doses cause neuroleptic effect. For its prokinetic effects it is used at very low doses. Hence it was considered to have a low potential to induce extrapyramidal side effects at the doses used in gastroenterology. But cases reported by Shin et al and Diwan et al showed that levosulpiride used at lower doses for prokinetic effects in elderly can cause frequent LIM.

Even though levosulpiride is excreted mainly via urine there is no recommendation to reduce its dose unlike its parent compound sulpiride in CKD patients on conservative management or hemodialysis. It is only partially removed by hemodialysis and its dialysability is unknown with continuous ambulatory peritoneal dialysis and continuous renal replacement therapies. In our study even though patients received the recommended dose of levosulpiride for prokinetic effects ( $25 \mathrm{mg}$ three times a day), they developed extrapyramidal symptoms rapidly after the administration of medication. Also they recovered quickly after the discontinuation of medication. The main stay of treatment in LIM is discontinuation of the drug. If dystonic, Parkinsonism and dyskinesia symptoms persist after discontinuing Levosulpiride, they should be treated with medications. Hence it appears that levosulpiride needs dose modification when used in patients with chronic kidney disease. Multi centric prospective studies in patients with various stages of CKD will throw light on spectrum of neurological complications with levosulpiride.

\section{Limitations Of The Study}

The study sample size is small. This study did not include all the patients with chronic kidney disease who received levosulpiride during the time period. It appears that the occurrence of LIM was very high in this study as there were no case reports in CKD patients in the literature (to best of our knowledge). The Author's colleagues did not experience many LIM cases in their practice; the reason could be low dose (25 mg / day) of levosulpiride being administered in their patients.

\section{Conclusion}

Levosulpiride is an effective prokinetic agent. Even though the occurrence of LIM is rare in patients with normal kidney function, LIM occurs much more commonly in chronic kidney disease patients contrary to the belief. Hence the dose of levosulpiride should be modified in patients with chronic kidney disease and prompt discontinuation of levosulpiride should be done with the occurrence of LIM as it leads to complete recovery of symptoms. More awareness and prompt recognition of these neurological complications by the primary care physicians is the key in the management. 
Patient Characteristics (Table 1)

\begin{tabular}{|c|c|c|c|c|c|c|c|c|c|c|c|c|}
\hline variables & pt1 & pt2 & pt3 & pt4 & pt5 & pt6 & pt7 & pt8 & pt9 & pt10 & pt11 & pt12 \\
\hline age & 66 & 35 & 22 & 36 & 30 & 70 & 50 & 55 & 25 & 35 & 53 & 28 \\
\hline diagnosis & $\begin{array}{l}\text { DM,HT, } \\
\text { CKD 5T }\end{array}$ & $\begin{array}{r}\mathrm{HT}, \\
\text { CKD 3T }\end{array}$ & $\begin{array}{r}\mathrm{HT}, \\
\text { CKD VD }\end{array}$ & $\begin{array}{r}\mathrm{HT}, \\
\text { CKD VD }\end{array}$ & CKD II & $\begin{array}{l}\text { DM, HT, } \\
\text { CKD III }\end{array}$ & $\begin{array}{l}\text { DM,HT, } \\
\text { CKD V }\end{array}$ & $\mathrm{HT}, \mathrm{CKD} V$ & $\begin{array}{l}\text { HT, CKD } \\
\text { VD }\end{array}$ & $\begin{array}{r}\mathrm{HT}, \\
\text { CKD VD }\end{array}$ & $\begin{array}{l}\text { DM,HT } \\
\text { CKD IV }\end{array}$ & $\begin{array}{r}\mathrm{HT}, \\
\text { CKD IV }\end{array}$ \\
\hline eGFR & 13 & 45 & 10 & 10 & 83 & 32 & 11 & 14 & 10 & 10 & 24 & 22 \\
\hline sex & $f$ & $\mathrm{~m}$ & $\mathrm{~m}$ & $\mathrm{~m}$ & $f$ & $f$ & $\mathrm{~m}$ & $\mathrm{~m}$ & $\mathrm{~m}$ & $\mathrm{~m}$ & $\mathrm{~m}$ & $\mathrm{~m}$ \\
\hline dose (mg/d) & 75 & 75 & 75 & 75 & 75 & 75 & 75 & 75 & 75 & 75 & 75 & 75 \\
\hline route & iv + oral & iv & oral & oral & oral & iv + oral & oral & oral & oral & oral & oral & iv \\
\hline s.k+ & $<3.5$ & $3.5-5$ & $>5 \mathrm{meq} / \mathrm{l}$ & $>5 \mathrm{meq} / \mathrm{l}$ & $3.5-5$ & $3.5-5$ & $>5 \mathrm{meq} / \mathrm{l}$ & $3.5-5$ & $>5 \mathrm{meq} / \mathrm{l}$ & $>5 \mathrm{meq} / \mathrm{l}$ & $3.5-5$ & $<3.5$ \\
\hline onset(days) & 10 & 2 & 5 & 3 & 3 & 21 & 21 & 3 & 2 & 3 & 4 & 2 \\
\hline $\begin{array}{c}\text { movement } \\
\text { disorder }\end{array}$ & $\begin{array}{l}\text { Orofacial } \\
\text { dyskinesia, } \\
\text { akathisia }\end{array}$ & dystonia & dystonia & dystonia & dystonia & Parkinson & Parkinson & dystonia & dystonia & dystonia & $\begin{array}{l}\text { tremor } \\
\mathrm{s}\end{array}$ & dystonia \\
\hline $\begin{array}{r}\text { duration } \\
\text { (days) }\end{array}$ & 7 & 3 & 3 & 3 & 3 & 90 & 36 & 2 & 3 & 4 & 3 & 2 \\
\hline outcome & recovered & recovered & recovered & recovered & recovered & lost & recovered & recovered & recover & recover & recover & recover \\
\hline
\end{tabular}

\section{References}

[1]. Gupta S, Garg GR, Halder S, Sharma KK. Levosulpiride: A Review. Delhi Psychiatry Journal. 2007;10(2):144-6.

[2]. Corazza GR, Biagi F, Albano O, Bianchi Porro G, Cheli R, Mazzacca G, et al. Levosulpiride in functional dyspepsia: a multicentric, double-blind, controlled trial. Ital J Gastroenterol. 1996;28(6):317-23.

[3]. Serra J. [Levosulpiride in the management of functional dyspepsia and delayed gastric emptying]. Gastroenterologia y hepatologia. 2010;33(8):586-90.

[4]. Shin H-W, Chung SJ. Drug-induced parkinsonism. Journal of clinical neurology. 2012;8(1):15-21.

[5]. Distrutti E, Fiorucci S, Hauer SK, Pensi MO, Vanasia M, Morelli A. Effect of acute and chronic levosulpiride administration on gastric tone and perception in functional dyspepsia. Alimentary Pharmacology \& Therapeutics. 2002;16(3):613-22.

[6]. Lee HC, Hwang SH, Kang SY. Levosulpiride-Associated Hemichorea. Yonsei Med J. 2016;57(3):803-5.

[7]. Shin HW, Kim MJ, Kim JS, Lee MC, Chung SJ. Levosulpiride-induced movement disorders. Movement Disorders. 2009;24(15):2249-53.

[8]. Diwan A, editor Levosulpiride induced Movement Disorder-A case series. Movement Disorders; 2015: WILEY-BLACKWELL 111 RIVER ST, HOBOKEN 07030-5774, NJ USA.

[9]. Tonini M, Cipollina L, Poluzzi E, Crema F, Corazza G, De Ponti F. Clinical implications of enteric and central D2 receptor blockade by antidopaminergic gastrointestinal prokinetics. Alimentary pharmacology \& therapeutics. 2004;19(4):379-90. 